\title{
Cyclosporin-associated akinetic mutism and extrapyramidal syndrome after liver transplantation
}

\author{
G L A Bird, J Meadows, J Goka, R Polson, Roger Williams
}

\begin{abstract}
Three patients developed akinetic mutism on the third day after the introduction of intravenous cyclosporin $A$, given for immunosuppression after liver transplantation. One patient in addition developed a florid orofacial dyskinesia, which resolved completely, as did the akinetic mutism, after withdrawal of cyclosporin. In these patients the time course of the akinetic mutism and extrapyramidal syndrome, which developed in the absence of any other identifiable cause, suggests cyclosporin A was the precipitating factor. Subsequently, two of the patients showed signs of pseudobulbar palsy with abnormalities in the pons on MRI scanning, suggesting central pontine myelinolysis (CPM). None of the patients had experienced significant fluctuations in serum sodium or other risk factors for central pontine myelinolysis and the exact relationship to the earlier cyclosporin related mutism was not clear.
\end{abstract}

Several reports have drawn attention to the wide range of neurological adverse effects attributable to cyclosporin A. These include the relatively common occurrence of tremor and seizures to the rarer cases of confusion, coma, ataxia, quadriparesis and cerebral blindness. ${ }^{1}$

In this report we detail the occurrence of akinetic mutism and severe dyskinesia, which were temporally related to the administration of cyclosporin. Two of the patients also subsequently developed pseudobulbar palsies which were much slower to resolve, and in these two cases the additional diagnosis of central pontine myelinolysis (CPM) was suspected.

\section{Case 1}

A 34 year old Japanese doctor had a liver transplantation in 1987 for decompensated cirrhosis secondary to chronic hepatitis $\mathbf{B}$. There was no history of neurological symptoms or previous neurological illness. Initial postoperative recovery was good and immunosuppression was given with prednisolone $(1 \mathrm{mg} / \mathrm{kg} /$ day $)$ and azathioprine
$(1.5 \mathrm{mg} / \mathrm{kg} /$ day). No anti-emetics were given. On the third postoperative day cyclosporin was started at a dose of $4 \mathrm{mg} / \mathrm{kg} /$ day, given as a twice daily intravenous infusion. Two days later the patient suddenly developed a trancelike state, becoming mute, with no spontaneous movement and unresponsive to all social stimuli. Neurological examination revealed oculogyric upward deviation of gaze, but no other neurological signs. Protective reflexes were maintained, but withdrawal to painful stimuli was sluggish.

Review of peri- and postoperative records showed normal serum glucose, electrolytes and urea, with serum sodium values of between 138 and $144 \mathrm{mmol} / \mathrm{l}$ (normal range 135$145 \mathrm{mmol} / \mathrm{l}$ ). Liver function tests indicated a well-functioning graft with a prothrombin time of $18 \mathrm{~s}$ (control 15s). Serum magnesium was $0.9 \mathrm{mmol} / 1$ (normal range $0.7-1.2 \mathrm{mmol} / \mathrm{l}$ ) and cholesterol $2.8 \mathrm{mmol} / 1$ (normal range 3.0 $7.0 \mathrm{mmol} / \mathrm{l}$ ). Microbiological investigations were negative. EEG showed no abnormality and a computerised tomography (CT) brain scan was also normal. Blood cyclosporin at this time (five hours after administration) was $646 \mathrm{ng} / \mathrm{ml}$ (polyclonal RIA, therapeutic range 200-800 ng/ml).

Cyclosporin was withdrawn and the patient made a full recovery after 48 hours. Cyclosporin was subsequently reintroduced at a smaller dose of $2 \mathrm{mg} / \mathrm{kg} /$ day after a further five days without adverse neurological effects and the patient was discharged home well four weeks later.

\section{Case 2}

A 48 year old Icelandic woman with poorly compensated alcoholic cirrhosis (abstinent for two years) when assessed for liver transplantation gave no history of neurological disease. A complete neurological examination was negative. Transplantation carried out in 1988 was straightforward, as was her immediate postoperative course. Immunosuppression was given with prednisolone $(1 \mathrm{mg} / \mathrm{kg} /$ day) and azathioprine $(1.5 \mathrm{mg} / \mathrm{kg} /$ day $)$ and on the third postoperative day cyclosporin was added at $2 \mathrm{mg} / \mathrm{kg} /$ day, given as a twice daily intravenous infusion. Two days later the trough cyclosporin level was $40 \mathrm{ng} / \mathrm{ml}$ (monoclonal RIA, therapeutic range $100-150 \mathrm{ng} / \mathrm{ml}$ ) and the dose was increased to $3 \mathrm{mg} / \mathrm{kg} /$ day. 
Figure 1 a, b: Grimacing and tongue protrusion with involuntary movements of upper limbs in case 2.

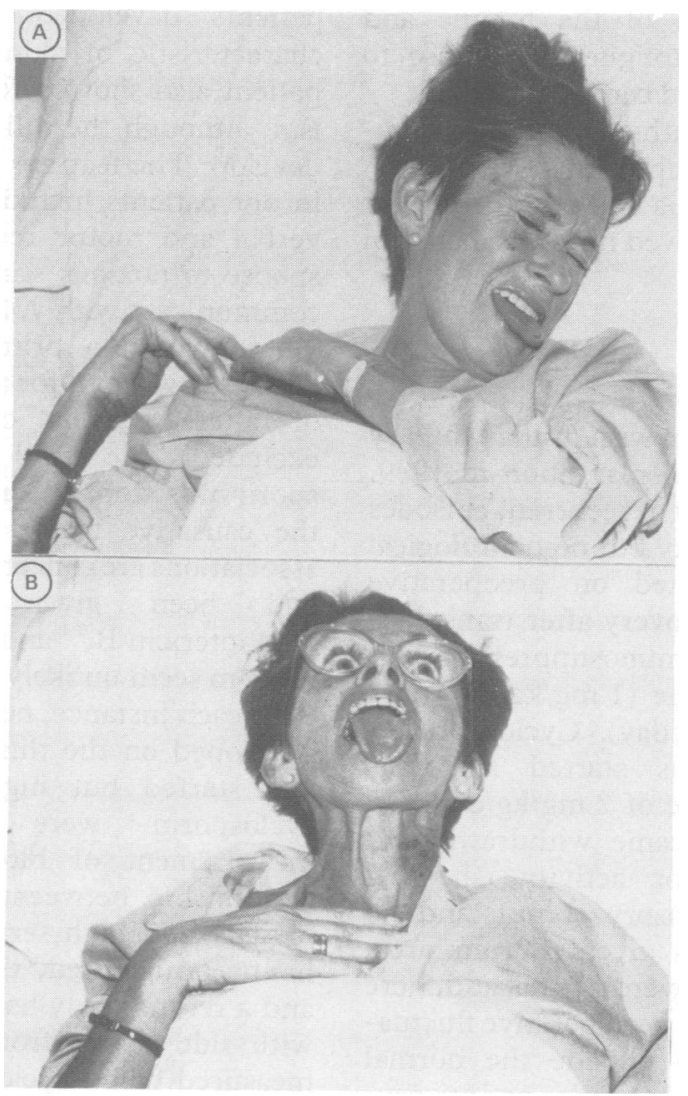

With the increased cyclosporin dose the patient gradually became mute and generally unresponsive over a period of three to four hours, with reduced spontaneous movement. Examination revealed a fine resting tremor and increased tone in all limbs. Shortly afterwards there was a two hour episode of severe orofacial dyskinesia with tongue protrusion and grimacing (fig 1) accompanied by choreoathetoid movements of the arms. This settled spontaneously, but within the next 12 hours the patient had three generalised seizures. Cyclosporin A was withdrawn and her neurological signs resolved over the next 48 hours. Seven days later she relapsed with trismus and an inability to swallow. Neurological assessment and indirect laryngoscopy revealed weakness but no spasticity of muscles innervated by the lower cranial nerves and screening of swallowing showed poor control of the oral phase but normal pharyngeal and oesophageal phases.

Examination of operative and perioperative records showed values for serum sodium of between 135 and $141 \mathrm{mmol} / 1$ and normal serum electrolytes, glucose and urea. Liver function tests revealed excellent graft function. No anti-emetics or anti-psychotic neuroleptics had been given. Serum cholesterol was $3.0 \mathrm{mmol} / 1$ and serum magnesium $0.58 \mathrm{mmol} / \mathrm{l}$. Correction of serum magnesium to within the normal range did not correlate with an improvement in the neurological signs. A second serum cyclosporin A level taken two hours after the onset of involuntary movements was $40 \mathrm{ng} / \mathrm{l}$ (monoclonal RIA). Cerebrospinal (CSF) fluid examination was normal with negative serology. EEG showed diffuse changes reported as being compatible with a metabolic abnormality. A CT brain scan showed mild atrophy in keeping with the previous alcohol abuse but no focal abnormalities. Magnetic resonance imaging (MRI) at 10 days showed a focal area of abnormality in the pons in T2 weighted images (fig 2a).

Over the next seven days she slowly improved, although requiring nasogastric feeding for several days. During this recovery period she developed a jaw jerk, but no spasticity of the bulbar muscles was observed. Cyclosporin A was reintroduced at the smaller dose of $1 \mathrm{mg} / \mathrm{kg} /$ day after seven days without ill effect. Her speech improved over the following three weeks, but on discharge, six weeks after grafting, she still had some
Figure 2 a: T2 weighted magnetic resonance image of case 2, 10 days after liver transplantation showing asymmetrical area of white matter abnormality in brainstem (arrowed; Gyrex S5000, 0.5 Tesla, TR2000

TE70). $b:$ T2 weighted magnetic resonance image of case 3 two weeks after transplantation, showing area of high signal intensity in mid-pons (arrowed; Picker 2055 HP, 1.5 Tesla, TR2000, TE80).
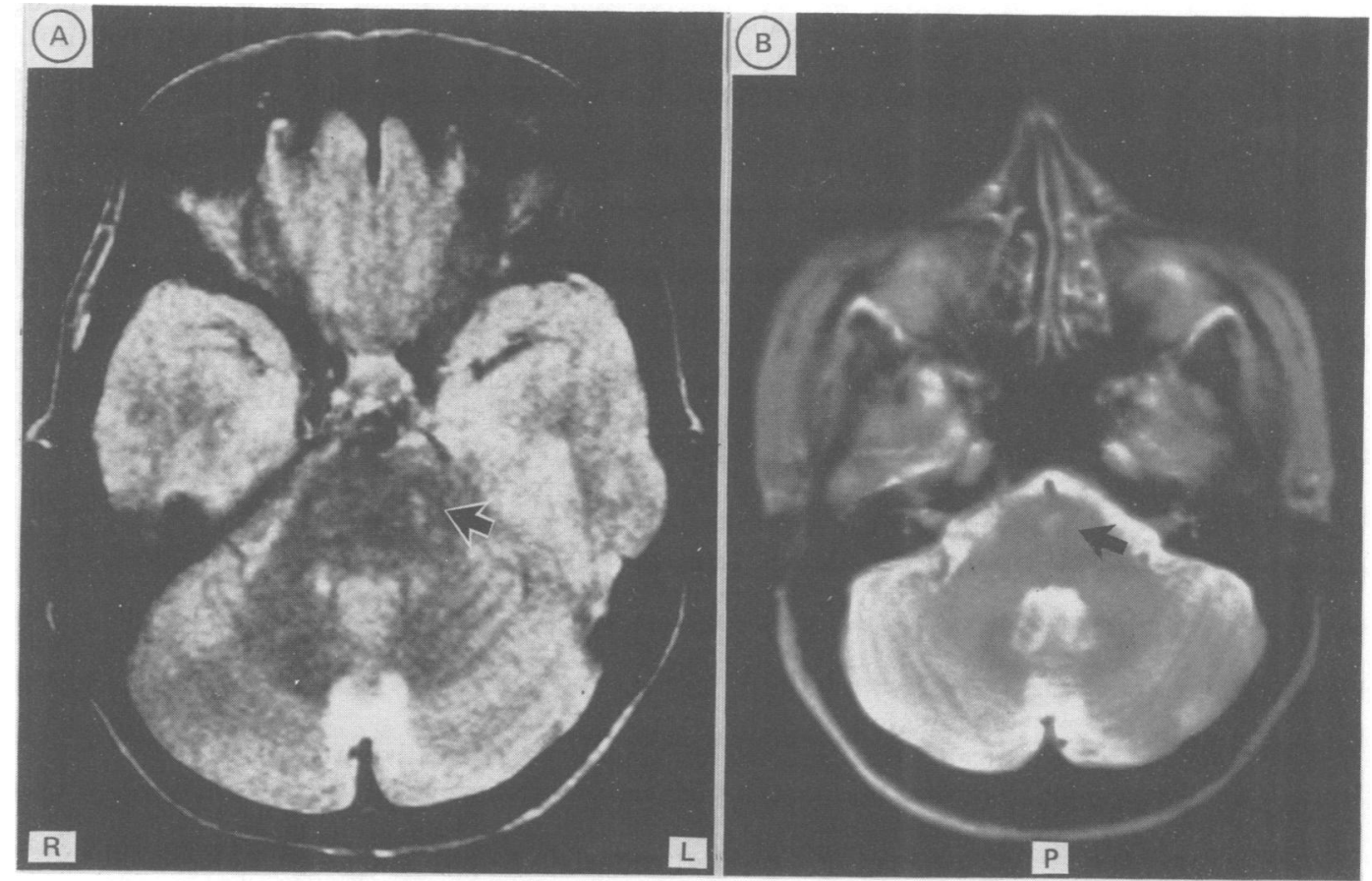
involuntary movements of the tongue and mild spasticity of the tongue giving rise to mild dysphonia, and mild cerebellar ataxia.

Reassessment 12 months after transplantation showed further improvement but with persistent mild dysphonia and mild cerebellar ataxia. Repeat MRI showed no abnormality in the pons.

\section{Case 3}

A 53 year old Irish woman with primary biliary cirrhosis had a liver transplantation in 1989. The patient had a history of recurrent episodes of hepatic encephalopathy but no neurological abnormality was detected on preoperative investigation. Initial recovery after transplantation was good and immunosuppression was started with prednisolone $(1 \mathrm{mg} / \mathrm{kg} /$ day $)$ and azathioprine $(1.5 \mathrm{mg} / \mathrm{kg} /$ day $)$. Cyclosporin $A$ immunosuppression was started six days postoperatively at a dose of $2 \mathrm{mg} / \mathrm{kg} /$ day. On the eighth day she became withdrawn and mute, with little motor activity, showing minimal reaction to sensory stimuli and no further cyclosporin was given. Serum urea, glucose and electrolytes were normal and there had been no operative or perioperative fluctuations in serum sodium outside the normal laboratory range. No anti-emetics or anti-psychotic neuroleptic drugs had been given. Over the next few days the patient became fully responsive to verbal and sensory stimuli, although there was in addition increasing difficulty in swallowing, requiring nasogastric feeding.

Neurological examination at the end of the second week after transplantation showed features of pseudobulbar palsy, with weakness and immobility of the tongue and palate causing severe dysphagia and dysarthria. Mild cerebellar ataxia and a jaw jerk were also present. Seven days later the jaw jerk and cerebellar ataxia had resolved and there were normal movements of the palate and tongue, although she remained dysarthric.

Liver function tests revealed good graft function. Serum cholesterol was $3.9 \mathrm{mmol} / 1$ and serum magnesium $0.65 \mathrm{mmol} / \mathrm{l}$, although correction to the normal range did not bring about an improvement. A random blood cyclosporin A level taken 24 hours after the first dose was $64 \mathrm{ng} / 1$ (monoclonal RIA). Examination of the CSF was normal and an EEG showed non-specific changes compatible with a metabolic abnormality. CT head scanning was normal. MRI showed a focal area of high signal intensity in the mid pons on T2 weighted images but was otherwise normal (fig 2b). Neurological symptoms resolved over the next four weeks and cyclosporin was reintroduced without any recurrence of symptoms.

\section{Discussion}

In all three patients the initial postoperative recovery was uncomplicated and neurological symptoms developed on the third day after cyclosporin had been introduced. Two of the patients developed a stuporose-like state characteristic of akinetic mutism; the third patient also showed features of akinetic mutism, although the full clinical picture did not develop. The features of akinetic mutism seen in our patients include a lack of spontaneous verbal and motor responses, a minimal response to noxious stimuli and an inability to communicate with voluntary eye movements. ${ }^{2}$ There were no primary motor or sensory deficits to account for akinesia and other causes of altered levels of consciousness were excluded. The rapid resolution when cyclosporin was stopped suggested cyclosporin was the causative factor. So far as other drug associations are concerned, none of our patients had been given methylphenidate or amphotericin $\mathrm{B}, 34$ and other causes of akinetic mutism seem unlikely. ${ }^{5}$

In each instance, neurological abnormalities developed on the third day after cyclosporin was started but high circulating levels of cyclosporin were not confirmed by measurement of blood levels. Although a relationship between central nervous system toxicity and high serum cyclosporin A levels has been suggested, ${ }^{6}$ this is not seen in all cases ${ }^{7}$ and a recent study has suggested that patients with side effects from cyclosporin in whom measured blood cyclosporin levels are within the therapeutic range have a genetically determined decrease of P450IIIa activity in the liver. ${ }^{8}$

According to a report from the Mayo Clinic, there is a correlation between cyclosporin neurotoxicity and low serum cholesterol levels in the first week after transplantation. ${ }^{9}$ Other investigators have postulated a role for hypomagnesaemia in CNS toxicity. ${ }^{10}$ In two of our patients mildly decreased serum magnesium levels were recorded, but there was no clear improvement following administration of magnesium. Serum cholesterol was decreased in one of the three patients only.

In the second case a florid orofacial dyskinesia developed although neuroleptic drugs and anti-emetics had not been administered. The patient also had seizures, one of the commoner neurological side effects of cyclosporin $\mathrm{A}$. The other neurological abnormality, a pseudobulbar palsy, seen in cases two and three appeared some time after withdrawal of cyclosporin $\mathrm{A}$ and after an improvement in mutism. In both patients, MRI revealed abnormalities in the pons, best seen in $\mathrm{T} 2$ weighted scans, compatible with CPM. ${ }^{11}$

In a recent study of CPM after liver transplantation, abnormalities of serum sodium were documented in all patients, yet in our patients there were no documented abnormalities or significant fluctuations of serum sodium, and other predisposing factors for CPM such as malnutrition, cerebrovascular insufficiency, infection, cerebral oedema, abnormalities in electrolytes and serum osmolality and liver dysfunction were not present. ${ }^{12}$ Although mutism is occasionally encountered as part of the clinical picture in CPM, it is usually associated with pyramidal tract signs and the "locked in" syndrome, in 
which the patient is able to communicate only by eye movements. ${ }^{5}$

In common with other reports of neurotoxicity attributed to cyclosporin A, rechallenge with cyclosporin was uneventful and in all three cases satisfactory oral immunosuppression was subsequently achieved based on a triple therapy regime.

We thank Professor Sir Roy Calne and Mr Peter Johnson, who performed the transplants, and also the many members of the then Hospital, and the Cromwell Hospital who contributed to the care of the patients.

1 Rubin AM, Kang H. Cerebral blindness and encephalopathy with cyclosporin A toxicity. Neurology 1987;37:1072-6.

2 Cairns HWB, Oldfield RC, Pennybacker JB, Whitteridge D. Akinetic mutism with an epidermoid cyst of the $3 \mathrm{rd}$ Akinetic mutism with an epide
ventricle. Brain 1941;64:273-91.
3 Wiener J, Kennedy S. Akinesia and mutism following a methylphenidate challenge test. J Clin Psychopharmacol 1985;5:231-3.

4 Devinsky O, Lemann W, Evans AC, Moeller JR, Rottenberg DA. Akinetic mutism in a bone marrow transplant recipient following total-body irradiation and amphotericin B chemoprophylaxis. Arch Neurol 1987;44:414-17.

5 Plum F, Posner JB. The diagnosis of stupor and coma, 3rd ed. Philadelphia: Davies, 1980.

6 Lavenstein B, Stewart C, Tina L. Cyclosporine-associated encephalopathy in childhood transplant patients. Trans Proc 1988;20:285-7.

7 Grigg MM, Costanzo Nordin MR, Celesia GG, et al. The aetiology of seizures after cardiac transplantation. Trans Proc 1988;20:937-44.

8 Lucey MR, Kolars JC, Merion RM, Campbell DA, Aldrich $M$, Watkins PB. Cyclosporin toxicity at therapeutic blood $M$, Watkins PB. Cyclosporin toxicity at therapeutic blood

9 De Groen PC, Aksamit AJ, Rakela J, Forbes GS, Kromm RAF. Central nervous system toxicity after liver transplantation: the role of cyclosporin and cholesterol. $N$ Eng J Med 1987;317:861-6

10 Thompson $\mathrm{CB}$, June $\mathrm{CH}$, Sullivan KM, Thomas ED Association between cyclosporin neurotoxicity and hypomagnesaemia. Lancet 1984;ii:1116-20.

11 Thompson PD, Miller D, Gledhill RF, Rossor MN Magnetic resonance imaging in central pontine myelinolysis Neurol Neurosurg Psychiatry 1989;52:675-7.

12 Estol CJ, Faris AA, Martinez J, Ahdab-Barmada M. Central pontine myelinolysis after liver transplantation. Neurology pontine myelinolys 